\title{
Small Extracellular Vesicles Derived from Adipose Tissue Prevent Bisphosphonate-Related Osteonecrosis of the Jaw by Promoting Angiogenesis
}

\author{
Jiao Huang ${ }^{1-4}$ \\ Lin Wang ${ }^{1-3}$ \\ Weidong $\operatorname{Tian}^{1-3}$ \\ 'State Key Laboratory of Oral Disease, \\ Engineering Research Center of Oral \\ Translational Medicine, Ministry of \\ Education, West China School of \\ Stomatology, Sichuan University, \\ Chengdu, Sichuan, People's Republic of \\ China; ${ }^{2}$ National Engineering Laboratory \\ for Oral Regenerative Medicine, Sichuan \\ University, Chengdu, Sichuan, People's \\ Republic of China; ${ }^{3}$ Department of Oral \\ and Maxillofacial Surgery, West China \\ Hospital of Stomatology, Sichuan \\ University, Chengdu, Sichuan, People's \\ Republic of China; ${ }^{4}$ West China Fourth \\ Hospital, Sichuan University, Chengdu, \\ Sichuan, People's Republic of China
}

Purpose: There is no definitive treatment for bisphosphonate-related osteonecrosis of the jaw (BRONJ). Small extracellular vesicles derived from adipose tissue (sEV-AT) have been proved efficient at promoting tissue regeneration. The aim of this study was to evaluate the effects of sEV-AT administration on BRONJ-like lesions in rats.

Methods: Zoledronate (Zol) and dexamethasone (Dex) were subcutaneously administered to create a BRONJ rat model. Rats were randomly divided into three groups: 1) Control; 2) Zol +Dex; 3) sEV-AT. The maxillary left first molars were extracted two weeks after the first administration. In the sEV-AT group, sEV-AT were given intravenously every three days right after tooth extraction. We preformed occlusal view images, microcomputed tomography $(\mu \mathrm{CT})$ and histological analysis to measure the regeneration of osseous and soft tissue in extraction sockets. Human umbilical vein endothelial cells (HUVECs) were isolated and cultured with endothelial cell medium (ECM). HUVECs were then divided into three groups: 1) Control: ECM; 2) Zol: ECM+Zol; 3) sEV-AT: ECM+Zol+sEV-AT. We evaluated the proliferation, tube formation and migration of HUVECs in each group.

Results: Rats treated with Zol+Dex showed BRONJ-like lesions including open wounds, necrotic bones, empty osteocyte lacunae and reduced osteoclasts. sEV-AT administration reduced BRONJ-like lesions by promoting soft tissue healing. $\mu \mathrm{CT}$ results showed that bone volume in extraction sockets in the sEV-AT group was larger than the Zol+Dex group. Histological analysis showed less necrotic bones and empty osteocyte lacunae in the sEV-AT group compared to the Zol+Dex group. Histological analysis also showed more osteoclasts, collagen fibers and blood vessels in the sEV-AT group compared to the Zol+Dex group. Furthermore, sEV-AT enhanced the proliferation, migration and tube formation of HUVECs which were inhibited by Zol.

Conclusion: Our findings indicate that sEV-AT prevent BRONJ in rats. Angiogenesis promotion contributes to the prevention of BRONJ.

Keywords: bisphosphonate-related osteonecrosis of the jaw, small extracellular vesicles, adipose tissue, endothelial cells, angiogenesis

\section{Introduction}

Bisphosphonates are widely used in the treatment of osteoporosis, multiple myeloma, breast cancers, and bone metastasis of cancer. Since first being reported by Marx in 2003, ${ }^{1}$ bisphosphonate-related osteonecrosis of the jaw (BRONJ), a severe side effect of bisphosphonates, has been a growing concern for oral and maxillofacial surgeons. ${ }^{2}$ BRONJ is defined as follows: 1) exposed bone in the maxillofacial region for longer than 8 weeks; 2) current or previous treatment with
Correspondence: Weidong Tian No. 14, 3rd Section of Renmin Road South, Chengdu, Sichuan, People's Republic of China

$\mathrm{Tel}+86 \quad 13608053216$

$\mathrm{Fax}+8602885503499$

Email drtwd@sina.com 
bisphosphonates; 3 ) no history of radiation therapy to the jaws or obvious metastatic disease to the jaws. ${ }^{3}$ Exposed necrotic bone in the oral cavity is a typical clinical manifestation of BRONJ, always accompanied by jaw pain and local soft swelling, leading to a marked decrease in patients' life quality. Although BRONJ has been studied over decades, the pathophysiology of the disease has not been fully elucidated. Several hypotheses try to explain its pathophysiology, such as disturbed bone remodeling, angiogenesis inhibition, inflammation and infection, soft tissue toxicity, immune dysfunction. ${ }^{4}$ The proper treatments of BRONJ are still under debate, and the efficacy is quite limited. 5,6

Adipose tissue, an energy storage depot, has been considered as a multifunctional organ that controls metabolic homeostasis, immunity, and satiety due to recent studies. $^{7,8}$ Adipose-derived stem cells (ADSCs) ${ }^{9,10}$ and stromal vascular fraction (SVF) cells ${ }^{11}$ of adipose tissue have been proved efficient at preventing BRONJ. However, cellular therapies may also be accompanied by some side effects, such as thromboembolism, protumorigenic, and undesired immune response. ${ }^{12,13}$ In addition, growing evidence has strongly indicated that most of the therapeutic efficacy of adipose tissue components is related to their paracrine activities. ${ }^{14}$ Small extracellular vesicles (sEV), which are lipid bilayer particles released from cells that consist of proteins, lipids, and RNA, play an essential role in inter-cellular communication. ${ }^{15}$ All cell types of adipose tissue, such as adipocytes, endothelial, immune cells and fibroblasts, contribute to the composition of small extracellular vesicles derived from adipose tissue (sEV-AT). Our previous studies suggest that sEV-AT can promote proliferation, migration, and angiogenic potential of endothelial cells and contribute to soft tissue regeneration. ${ }^{16,17}$ However, the therapy potential of sEV-AT requires further study.

We hypothesized that sEV-AT transplantation could prevent the development of BRONJ by promoting angiogenesis. In this study, we evaluated the effects of sEV-AT injection on tooth extraction socket healing in a rat BRONJ model.

\section{Materials and Methods}

\section{Animals}

Animals were obtained from Dashuo Experimental Animal Co. Ltd. (Chengdu, China). This study was reviewed and approved by the Ethics Committees of the State Key
Laboratory of Oral Diseases, West China School of Stomatology, Sichuan University. The approval number is WCHSIRB-D-2021-028. The care and use of the laboratory animals followed the guidelines of the Institutional Animal Care and Use Committee of West China School of Stomatology, Sichuan University.

\section{Preparation of sEV-AT}

SEV-AT were prepared as previously described. ${ }^{16,17}$ Fat pads were isolated from 4-week-old SD rats. Minced fat pieces were cultured with Serum-free $\alpha$-modified Eagle's medium ( $\alpha$-MEM, HyClone, Utah, USA) in a Celstir spinner flask (Wheaton, USA) at $100 \mathrm{rpm}, 37^{\circ} \mathrm{C}$, in $5 \% \mathrm{CO}_{2}$ for $48 \mathrm{~h}$. After removing tissue pieces by gauze and cellular debris by centrifugation $\left(2000 \mathrm{~g}, 4^{\circ} \mathrm{C}, 20 \mathrm{~min}\right)$, the supernatant was filtered through a $40 \mu \mathrm{m}$ filter (Corning, NY, USA) to get adipose tissue extract (ATE). ATE was filtered through $0.22 \mu \mathrm{m}$ filters (Millipore, Cork, Ireland), then concentrated by Ultracel-3 membrane (Millipore, Cork, Ireland) at $5000 \mathrm{~g}, 4^{\circ} \mathrm{C}$ for $30 \mathrm{~min}$, followed by further concentration by Ultracel-100 membrane (Millipore, Cork, Ireland) at $5000 \mathrm{~g}, 4^{\circ} \mathrm{C}$ for $30 \mathrm{~min}$. The concentrated ATE was mixed with the Total Exosome Isolation TM reagent (Invitrogen, Vilnius, Lithuania) at $4^{\circ} \mathrm{C}$ overnight and spun down at $10,000 \mathrm{~g}, 4^{\circ} \mathrm{C}$ for $1 \mathrm{~h}$ to obtain sEV-AT. sEV-AT were measured in terms of total protein amount determined by the bicinchoninic protein assay method according to the manufacturer's protocol (BCA Protein Assay Kit, KeyGEN BioTECH, Nanjing, China).

\section{Analysis of sEV-AT}

The isolated sEV-AT were visualized using a transmission electron microscope (TEM, Tecnai G2 F20 S-TWIN, FEI, Oregon, USA) by negative staining. The particle size and size distribution of sEV-AT were determined by ZetaVIEW S/N 19-480 analysis system (Software ZetaView version 8.05.11, Particle Metrix, Meerbusch, Germany) according to the manufacturer's protocol. The protein markers (CD63, CD9, HSP70 and actin) were detected by Western blotting. sEV-AT were labeled with membrane-labeling dye $\mathrm{DiO}$ (Invitrogen) in serum-free $\alpha-\mathrm{MEM}$ at $37^{\circ} \mathrm{C}$ for $20 \mathrm{~min}$. Then, DiO-labeled sEV-AT were re-purified with the Total Exosome Isolation TM reagent. HUVECs were co-cultured with DiO-labeled sEV-AT for 6 hours, washed with PBS, fixed in 4\% paraformaldehyde, stained with phalloidin (Invitrogen) and DAPI, washed with PBS and imaged by confocal microscopy (FV1000, Olympus, Tokyo, Japan). 


\section{In vivo Biodistribution of sEV-AT}

sEV-AT were mixed with DiR (PerkinElmer, USA) dye in serum-free $\alpha$-MEM at $37^{\circ} \mathrm{C}$ for $20 \mathrm{~min}$. Then, DiR-labeled sEV-AT were re-purified with the Total Exosome Isolation TM reagent. DiR-labeled sEV-AT were injected into SD rats' tail vein immediately after the maxillary left the first molar extraction. DiR fluorescence was analyzed using the Maestro EX pro in vivo imaging system (PerkinElmer, USA) at 1, 12, 24 hours post-injection. DiR fluorescence in brain, maxilla, lung, heart, liver, spleen and kidney was also measured.

\section{Animal Model}

Thirty-five 8-week-old female SD rats $(200 \pm 10 \mathrm{~g})$ were used in this study. Rats were randomly divided into three groups: 1) Control ( $\mathrm{n}=7)$; 2) Zol+Dex ( $=14)$; 3) sEV-AT $(\mathrm{n}=14)$. To create a rat BRONJ model, both Zol $(66 \mu \mathrm{g} /$ kg, Chiataitianqing Pharma, Jiangsu, China) and Dex (5 mg/kg, Quanyu Pharma, Shanghai, China) were subcutaneously administered three times per week for 4 weeks according to previous reports. ${ }^{18}$ Two weeks after the first administration, the maxillary left first molars were extracted with general and local anesthesia. sEVAT $(1 \mu \mathrm{g} / \mathrm{kg})$ were injected into the tail vein every three days after tooth extraction in the sEV-AT group. Equal volume of saline was injected in the Zol+Dex group. Untreated rats were euthanized at 2 weeks postextraction as natural healing control. Rats were euthanized at 2 weeks ( $\mathrm{n}=7$ each group) and 4 weeks ( $\mathrm{n}=7$ each group) post-extraction in the other two groups.

\section{Evaluation of Gross Wound Healing of Tooth Extraction Sockets}

Occlusal view images of tooth extraction sockets were taken after euthanasia with stereo microscope (SZX2-ILLT, Olympus, Tokyo, Japan). Borderlines between epithelium and exposed bone were digitally drawn using NIH ImageJ (https://imagej.nih.gov/ij/). The area surrounded by the borderline was defined as a wound open area.

\section{Microcomputed Tomography $(\mu \mathrm{CT})$ Assessment}

Left maxillae were dissected, fixed with $4 \%$ of paraformaldehyde and scanned by $\mu \mathrm{CT} 50$ (SCANCO Medical AG, Zurich, Switzerland) using a voxel resolution and an energy level of $10 \mu \mathrm{m}$ and $70 \mathrm{kV}$, respectively. Threedimensional (3D) images were reconstituted using software SCANCO Visualizer 1.1.18.0. Bone morphometric analysis of the extraction sockets was performed using the software SCANCO Evaluation 1.1.19.0. For bone morphometric analysis, Bone volume/Total volume (BV/TV), trabecular number (Tb.N), trabecular thickness (Tb.Th) and trabecular separation (Tb.Sp) were measured.

\section{Histological Analysis}

After radiological assessment, the samples were decalcified with $10 \%$ ethylenediaminetetraacetic acid (EDTA) at $4^{\circ} \mathrm{C}$ for 2 weeks. Demineralized maxillae were paraffinembedded and sectioned at a thickness of $6 \mu \mathrm{m}$. Hematoxylin and eosin (HE, Solarbio, Beijing, China) staining was carried out. Bone portion which equals to or is greater than 10 adjacent empty osteocyte lacunae is defined as necrotic bone. ${ }^{19}$ Empty osteocyte lacunae were counted in tooth extraction sockets and expressed as a percentage of total bone lacunae (\%). Tartrateresistant acid phosphatase (TRAP, Fujifilm, Osaka, Japan) staining was carried out to detect TRAP positive cells per linear bone perimeter $(\# / \mathrm{mm})$. Masson's trichrome (Baso, Zhuhai, China) staining was performed to evaluate collagen fibers. The content of VEGFA was detected by immunohistochemical (IHC) staining. After deparaffinization, hydration and blockage of endogenous peroxidase, the sections were incubated for $1 \mathrm{~h}$ with normal goat serum in order to block specific sites and then incubated overnight at $4{ }^{\circ} \mathrm{C}$ with primary anti-VEGFA antibody (Abcam, ab46154, USA). The secondary antibody was shown by the DAB kit (Gene Tech, Shanghai, China). The images were analyzed using NIH ImageJ.

\section{HUVECs Isolation and Culture}

Human umbilical cords were obtained from the Department of Obstetrics of the West China Second University Hospital of Sichuan University. The approval number is WCHSIRB-D-2021-015. We obtained the signed informed consent from the parents, in accordance with the Declaration of Helsinki. HUVECs were isolated from the umbilical vein as previously reported with some modification. ${ }^{20}$ First, the collected umbilical cords were rinsed twice with phosphate-buffered saline. Then, the umbilical vein was filled with $0.2 \%$ collagenase and incubated for $30 \mathrm{~min}$ in a humidified atmosphere of $37^{\circ} \mathrm{C}$ and $5 \% \mathrm{CO}_{2}$ for isolation of HUVECs. After that, the cells were collected and cultured in $25 \mathrm{~cm}^{2}$ tissue culture flasks (Corning) with ECM (ScienCell, California, USA). 


\section{Cell Proliferation Assay}

HUVECs were seeded onto 96-well plates (NEST, Jiangsu, China) at 500 cells per well. After $24 \mathrm{~h}$, the culture medium was replaced with ECM $(100 \mu \mathrm{L})$ containing 0 , 5, 10, 15, 20 uM Zol (Sigma-Aldrich, USA) respectively. The cell number was evaluated using the cell-counting kit8 (CCK8, KeyGEN BioTECH, Nanjing, China) according to the manufacturer's instructions. Growth curves were drawn with the absorbance values $(\mathrm{n}=5)$. Then, HUVECs were divided into three groups: (1) Control: HUVECs cultured with ECM, (2) Zol: HUVECs cultured with ECM and Zol, (3) sEV-AT: HUVECs cultured with ECM, Zol and sEV-AT $(50 \mu \mathrm{g} / \mathrm{mL})$. Growth curves were drawn for each group using the same method.

\section{Tube Formation Assay}

HUVECs $\left(8 \times 10^{3}\right)$, suspended with $50 \mu \mathrm{L}$ medium as the group division described above, were seeded onto angiogenesis u-slide (Ibidi, Gräfelfing, Germany) coated with Matrigel (10 ul, Corning, USA). After incubation for $4 \mathrm{~h}$, phase-contrast images were captured by an inverted microscope (Olympus, TH4-200, Tokyo, Japan). Total length and total nodes were measured using angiogenesis analyzer of ImageJ.

\section{Cell Migration Assay}

Cell migration was measured using a $6.5 \mathrm{~mm}$ Transwell $^{\circledR}$ with $8.0 \mu \mathrm{m}$ Pore Polycarbonate Membrane Insert (Corning, USA). In the upper chamber, HUVECs were added at a density of $2 \times 10^{4}$ cells per well. In the lower chamber, $600 \mu \mathrm{L}$ medium as the group division described above were added and then incubated for $18 \mathrm{~h}$. Cells that migrated to the lower surface of the membrane were fixed with $4 \%$ paraformaldehyde and stained with crystal violet staining solution (Solarbio, Beijing, China). The number of migrated cells was counted in three randomly selected microscopic fields. All in vitro experiments were carried out with three independent replications.

\section{Statistical Analysis}

Results are expressed as mean value-standard deviation. An unpaired 2-tailed Student's $t$ test was applied when comparing 2 groups. To analyze 3 or more independent groups, we used a 1-way analysis of variance (ANOVA), followed by Tukey's post hoc test. If the two-tailed $\mathrm{P}$ value was $<0.05(*),<0.01(* *),<0.001(* * *)$, it can be considered that the data were significantly different.

\section{Results}

\section{Characterization of sEV-AT and Cellular Uptake}

sEV were isolated from rat adipose tissue using a kit-based ultrafiltration method we have previously reported. ${ }^{16,17}$ Transmission electron microscopy analysis confirmed that sEV-AT were round-shaped vesicles surrounded by a bilayer membrane (Figure 1A). Nanoparticle tracking analysis by ZataView confirmed the size and its distribution. The sEVAT had various sizes with a peak at $122 \mathrm{~nm}$ (Figure 1B). Western blot analysis confirmed the presence of exosomal protein markers (CD9, CD63, and HSP70) in SEV-AT. And the cellular protein actin was not detected on the contrary (Figure 1C). Furthermore, we confirmed that the sEV-AT labeled with $\mathrm{DiO}$ were taken into the cell cytoplasm of HUVECs when cultured together (Figure 1D).

\section{Biodistribution of sEV-AT}

Non-invasive in vivo imaging showed that DiR-labeled sEV-AT predominantly accumulated in the liver at 1 $\mathrm{h}$ post-injection. At $12 \mathrm{~h}$ and $24 \mathrm{~h}$ post-injection, a clear distribution profile of liver and spleen was detected (Supplementary Figure S1A). Furthermore, we performed ex vivo imaging of major organs to find the complete biodistribution pattern of EVs-AT (Supplementary Figure $\underline{\mathrm{S} 1 \mathrm{~B}}$. . The signals from the maxilla were also successfully detected at $12 \mathrm{~h}$ post-injection, which was mainly concentrated in the tooth extraction socket. At $24 \mathrm{~h}$ post-injection, the intensity of signals increased, and the range of signals was wider (Figure 2).

\section{Clinical Effects of sEV-AT on Wound Healing of Tooth Extraction Sockets}

Zol, Dex and sEV-AT were injected according to the schedule (Figure 3A). Two weeks after tooth extraction, the Zol+Dex group and sEV-AT group showed exposed bone without soft tissue coverage, whereas extraction sockets were healed in the control group (Figure 3B). However, sEV-AT therapy significantly decreased the wound open area $(p<0.001)$ (Figure $3 \mathrm{C})$. Four weeks after tooth extraction, wound open area with exposed bone was observed in the Zol+Dex group, which confirming the establishment of rat BRONJ model. In contrast, the sEV-AT group showed wound healing in most cases (5/7) (Figure 3B and C).

$\mu \mathrm{CT}$ analysis showed that extraction sockets were filled with newly formed bones in the control group. On the 
A
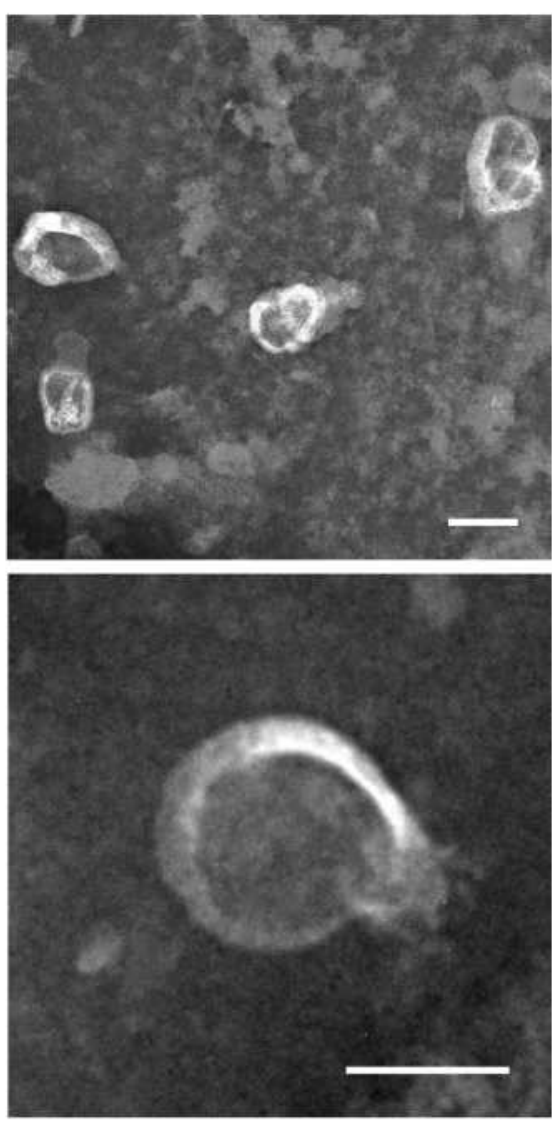

C

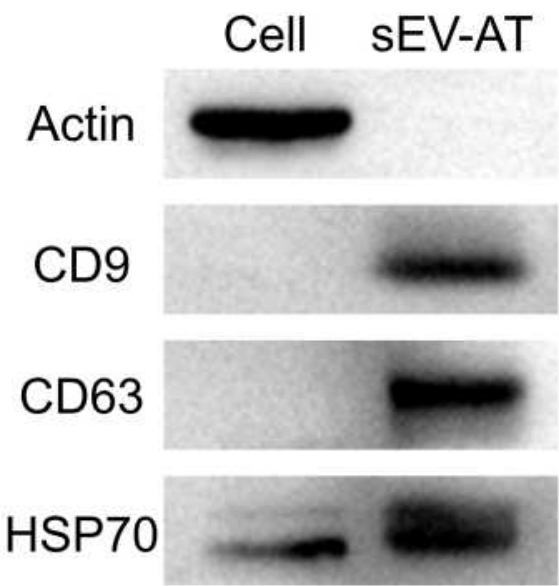

B

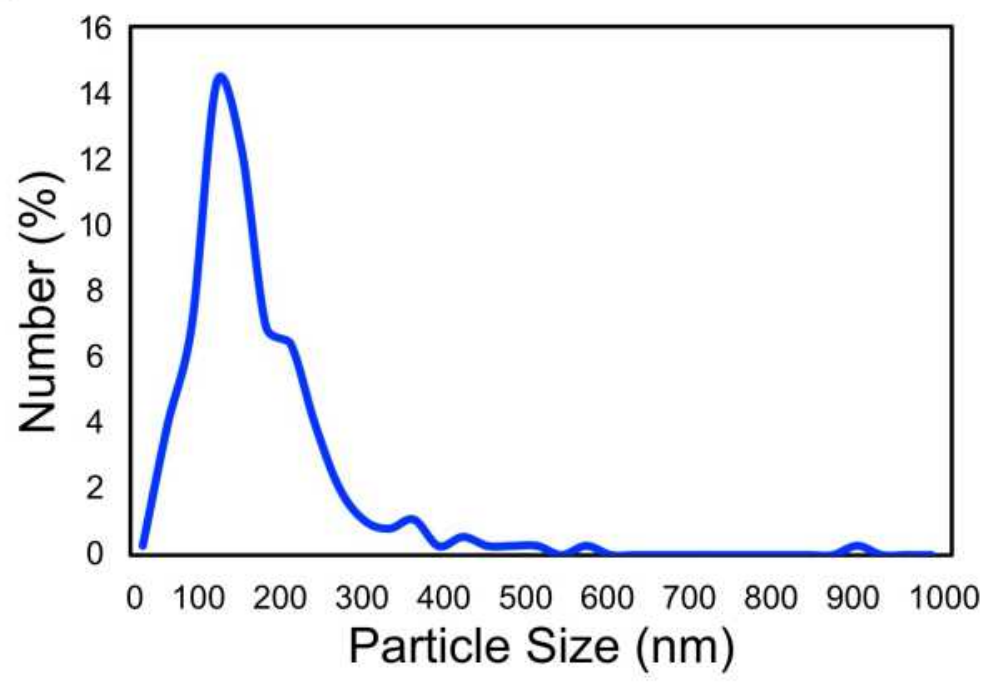

D
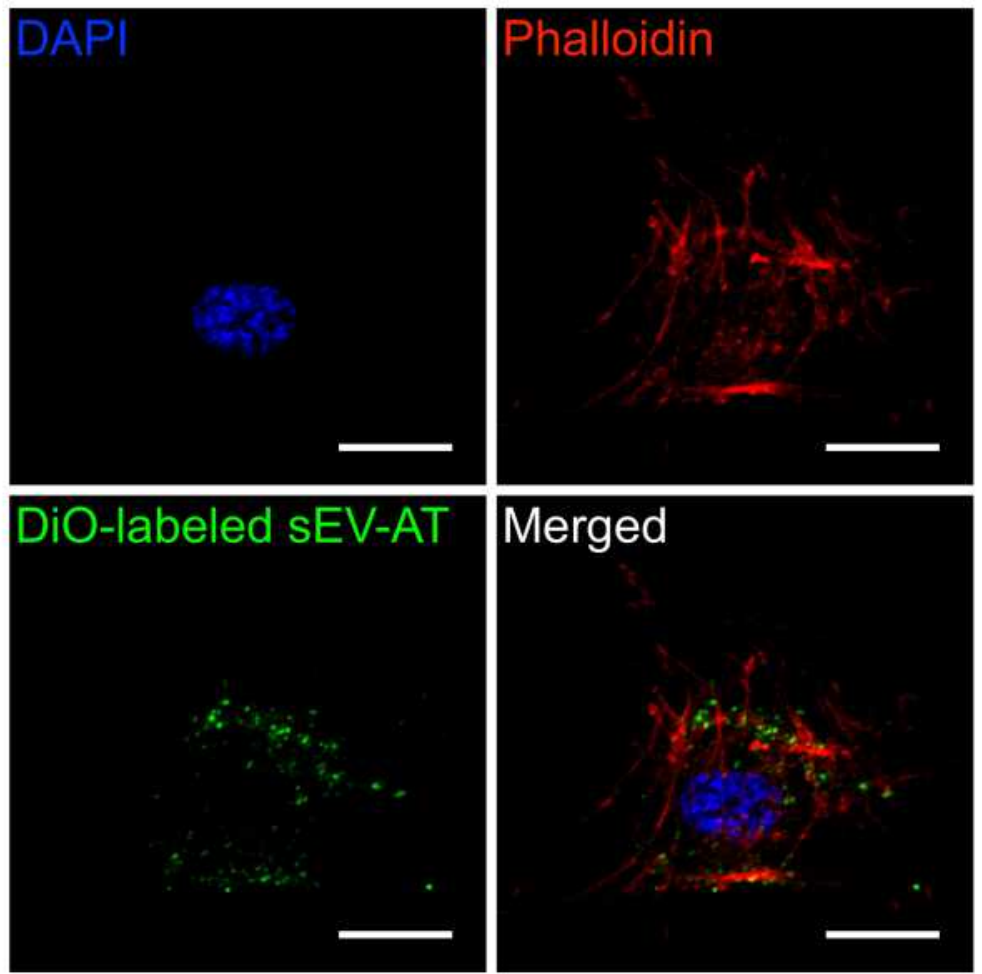

Figure I Characterization of sEV-AT. (A) Representative images of sEV-AT with transmission electron microscopy. Scale bar=I00 nm. (B) The particle size distribution of sEV-AT was measured by ZataView analysis. (C) Western blot analysis of exosomal markers, CD63, CD9, and HSP70. Actin was cellular protein as a control. (D) Uptake analysis of sEV-AT by HUVECs (red: phalloidin, green: DiO-labeled sEV-AT, blue: nuclei). Scale bar $=20 \mu \mathrm{m}$.

Abbreviations: sEV-AT, small extracellular vesicles derived from adipose tissue. HSP70, heat shock protein 70. HUVECs, human umbilical vein endothelial cells.

contrary, extraction sockets were almost empty in the Zol + Dex group. Bone volume in extraction sockets appeared to be larger in the sEV-AT group than the Zol+Dex group
(Figure 3D). Bone morphometric analysis showed that sEV-AT treatment significantly increased BV/TV, Tb.N, Tb.Th, and decreased Tb.Sp $(\mathrm{p}<0.001)$ (Figure 3E). 


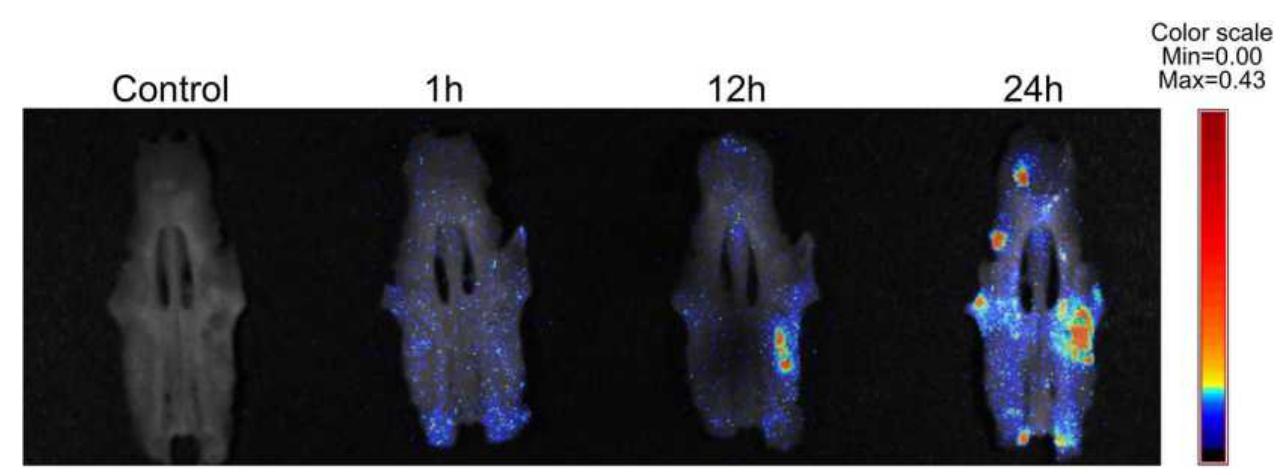

Figure 2 Biodistribution of DiR-labeled sEV-AT to maxilla. Ex vivo images of maxillae from rats after intravenous injection of DiR-labeled sEV-AT. Abbreviation: sEV-AT, small extracellular vesicles derived from adipose tissue.

\section{Histological Effects of sEV-AT on Tooth Extraction Sockets}

Histological analysis was performed to further investigate how sEV-AT work on wound healing in tooth extraction sockets. Zol+Dex treatment led to necrotic bones and empty osteocyte lacunae. Necrotic bones in the sEV-AT group were less and smaller than the Zol+Dex group (Figure 4A). Furthermore, the percentage of empty osteocyte lacunae was reduced with the administration of sEVAT $(p<0.001)$ (Figure 4B). TRAP staining showed that the average number of osteoclasts per linear bone perimeter was significantly reduced in the Zol+Dex group compared to the control group $(p<0.001)$. However, sEV-AT significantly increased the average number of osteoclasts $(p<0.001)$ (Figure 4C and D). Masson's trichrome staining showed that sEV-AT significantly increased the production of collagen fibers $(\mathrm{p}<0.001)$ (Figure $4 \mathrm{E}$ and $\mathrm{F}$ ). Immunohistochemical staining showed that Zol+Dex treatment reduced the number of blood vessels, whereas sEVAT therapy led to an appreciable increase in the number of blood vessels $(\mathrm{p}<0.001)$ (Figure $4 \mathrm{G}$ and $\mathrm{H}$ ).

\section{sEV-AT Promoted the Proliferation, Migration and Tube Formation of HUVECs Which Were Inhibited by Zol}

To explore the effects of Zol on cell proliferation, $5 \mu \mathrm{M}$, $10 \mu \mathrm{M}, 15 \mu \mathrm{M}, 20 \mu \mathrm{M}$ Zol were treated with HUVECs. The results showed that Zol obviously inhibited the proliferation of HUVECs, and the higher the concentration was, the stronger the inhibitive effect was (Figure 5A). Based on the results, $10 \mu \mathrm{M}$, the lowest concentration that had a statistically negative effect on HUVECs proliferation, was chosen for the rest of the study. The results of
CCK8 showed that sEV-AT could reverse the inhibiting effect of Zol on HUVECs proliferation (Figure 5B). Tube formation assay showed that Zol inhibited the formation of tube-like structures. The total length and total nodes of HUVECs were reduced after Zol stimulating $(\mathrm{p}<0.01)$. However, sEV-AT could promote the tube formation of HUVECs in the Zol-stimulated environment, represented as the increasing of the total length $(\mathrm{p}<0.05)$ and total nodes $(\mathrm{p}<0.01)$ (Figure $5 \mathrm{C})$. Transwell migration assay showed that Zol inhibited the migration of HUVECs $(p<0.01)$. sEV-AT promoted the migration of HUVECs in the Zol-stimulated environment $(\mathrm{p}<0.05)$ (Figure 5D).

\section{Discussion}

The risk of BRONJ in patients who have received zoledronate is higher than those treated with other bisphosphonates. $^{21,22}$ The risk of BRONJ is increased by multiple immunosuppressive drugs, such as corticosteroids and chemotherapeutic agents, which are frequently used in cancer therapy. ${ }^{23,24}$ In addition, tooth extraction has been proved to be a major local risk factor for BRONJ. ${ }^{25}$ According to these clinical research findings, BRONJlike animal models were built via combining the administration of zoledronate and dexamethasone, along with tooth extraction. ${ }^{18,26,27}$ In our study, we built the BRONJ rat model using the method reported by Kaibuchi N, ${ }^{18}$ which stated that BRONJ-like lesions were observed in all cases. The method was proved to be efficient that all rats in the Zol+Dex group have developed BRONJ-like lesions. The American Association of Oral and Maxillofacial Surgeons defines BRONJ as exposed bone or bone that can be probed through an intraoral or extraoral fistula in the maxillofacial region which has persisted for longer than 8 weeks. ${ }^{3}$ However, extraction sockets in 
A

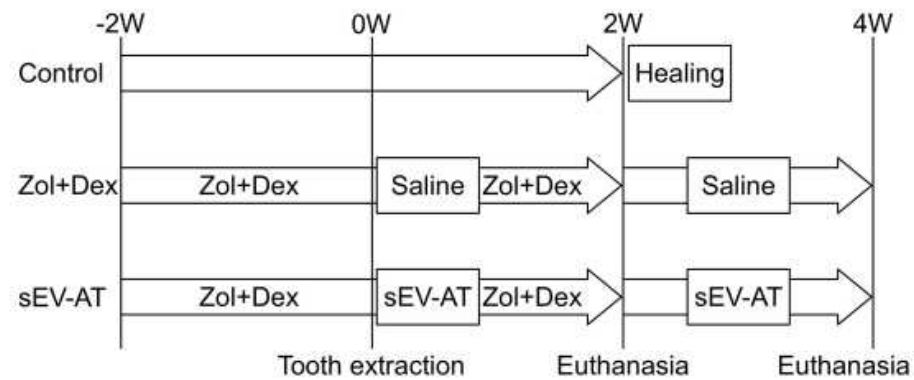

C

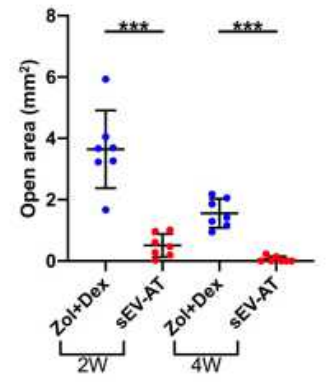

B

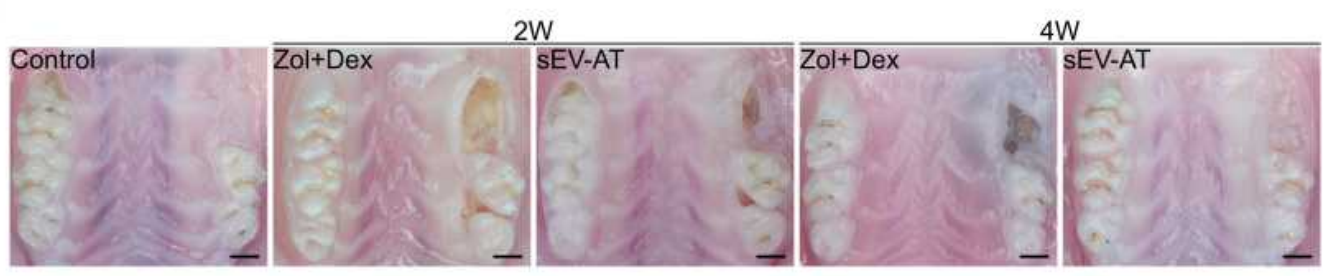

D

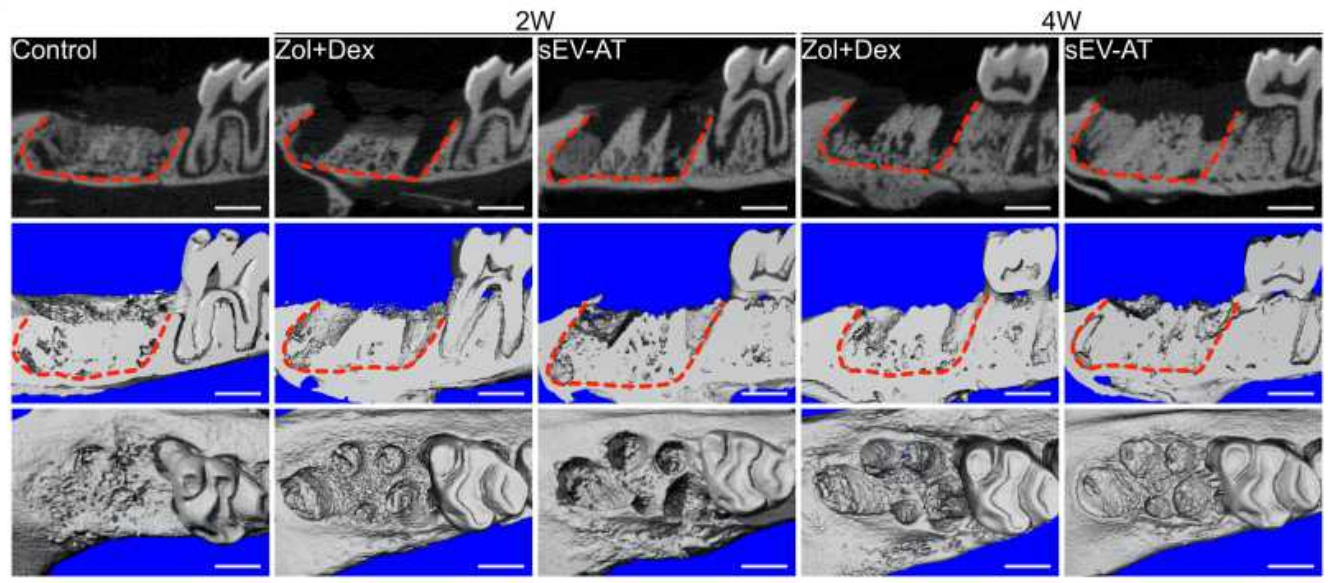

$\mathbf{E}$

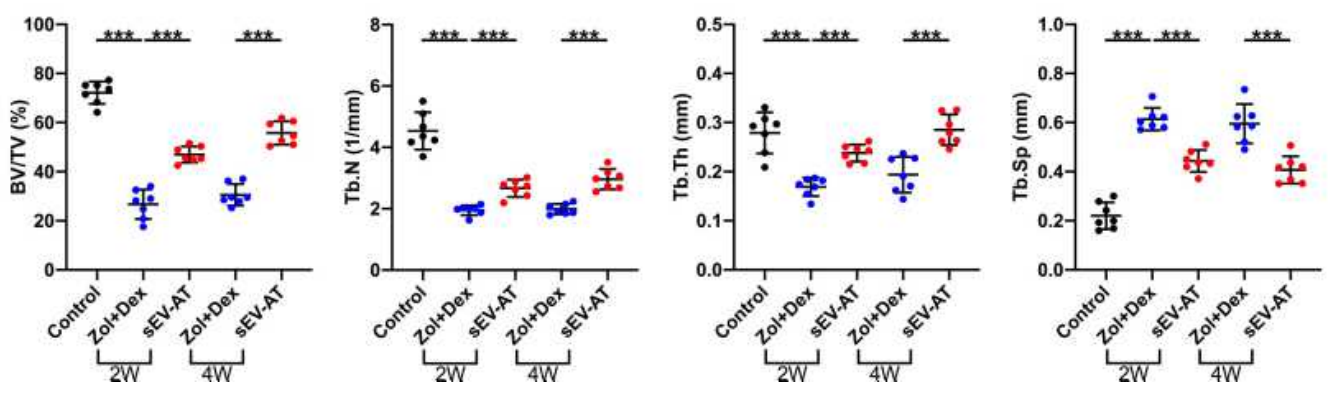

Figure 3 Effects of sEV-AT injection on rat BRONJ model. (A) Development of rat BRONJ model and schedule of sEV-AT injection (Control: natural healing group; Zol +Dex: Zol+Dex and saline treated group; sEV-AT: Zol+Dex and sEV-AT treated group). (B) Representative intraoral photos. Scale bar=Imm. (C) Open area without epithelium coverage. Open area was significantly decreased by sEV-AT treatment (***p<0.00I). (D) Representative $\mu C T$ images of tooth extraction sockets (red dotted lines: tooth extraction sockets). Scale bar=Imm. (E) Quantification of BV/TV, Tb.N, Tb.Th and Tb.Sp in each group $(* * * p<0.00 I)$.

Abbreviations: sEV-AT, small extracellular vesicles derived from adipose tissue. BRONJ, bisphosphonate-related osteonecrosis of the jaw. $\mu C T$, microcomputed tomography. BV/TV, bone volume/total volume. Tb.N, trabecular number. Tb.Th, trabecular thickness. Tb.Sp, trabecular separation. Zol, zoledronate. Dex, dexamethasone. 
A

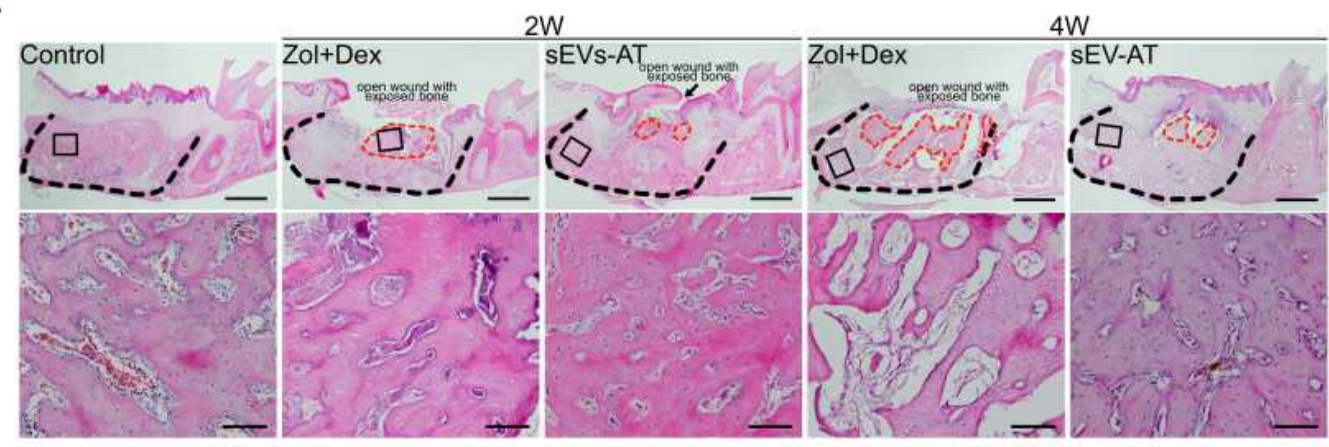

C

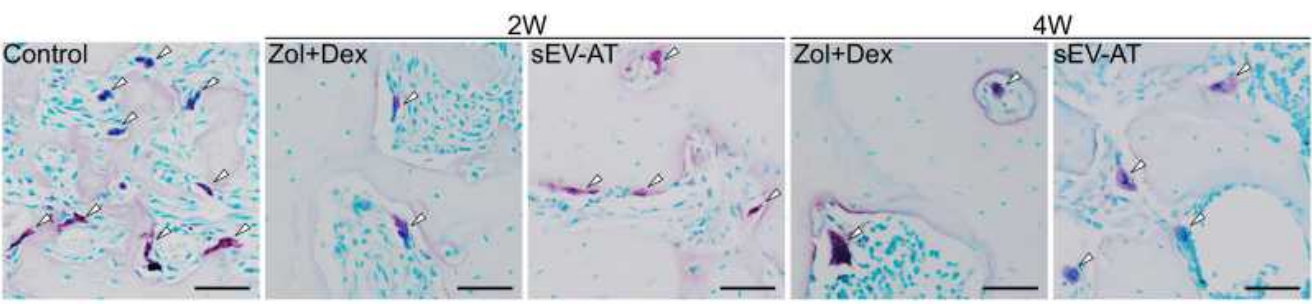

E
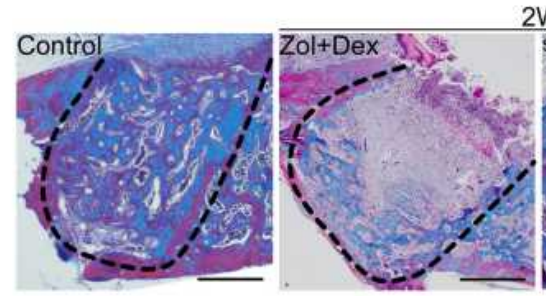

2W

$4 \mathrm{~W}$
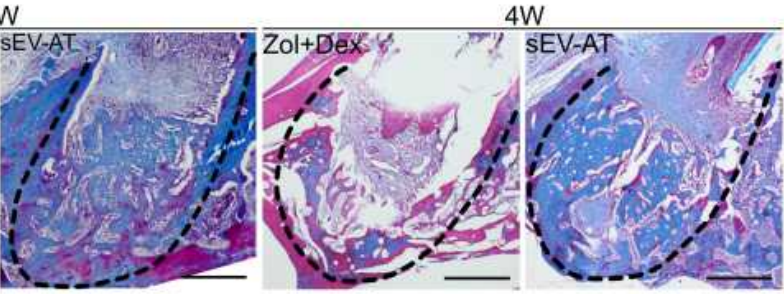

G
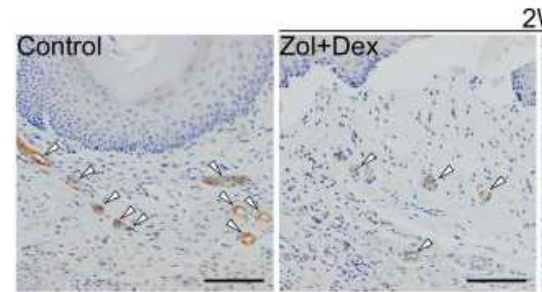

$2 W$
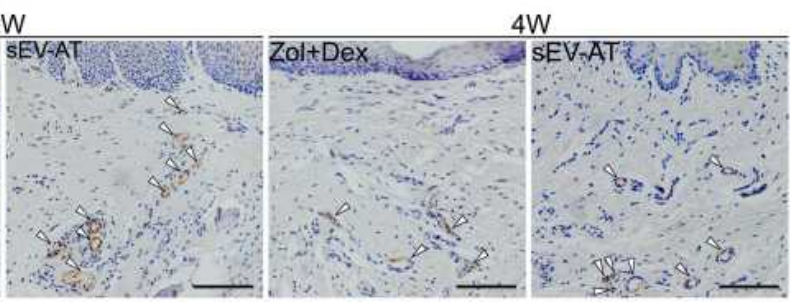

B

D

F
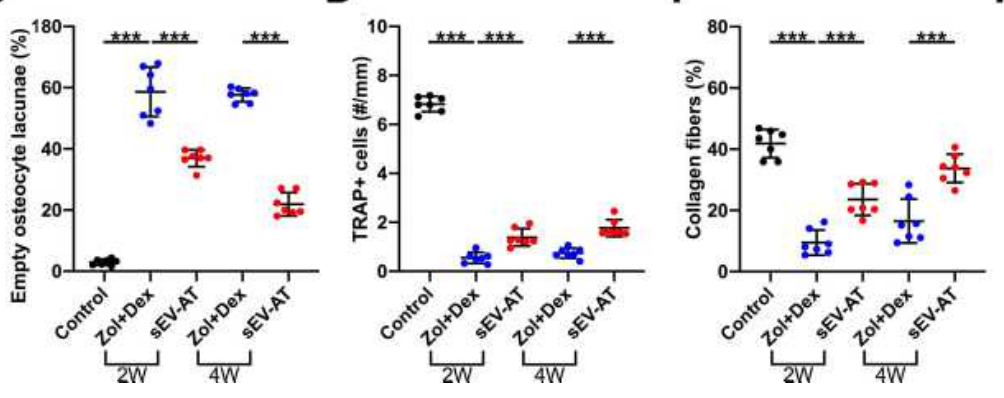

H

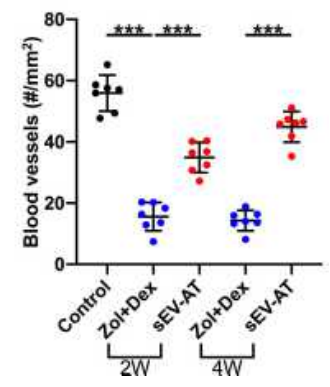

Figure 4 Histological analysis of tooth extraction sockets in each group (Control: natural healing group; Zol+Dex: Zol+Dex and saline treated group; sEV-AT: Zol+Dex and sEV-AT treated group). (A) Representative HE-stained images of tooth extraction sockets (black dotted line: tooth extraction sockets, red dotted line: necrotic bones, black square: areas were magnified). Scale bar $=1 \mathrm{~mm}$ (upper), scale bar $=100 \mu \mathrm{m}$ (lower). (B) The percentage of empty osteocyte lacunae $(* * * p<0.00 \mathrm{I})$. (C) Representative TRAP-stained images of tooth extraction sockets (white arrowhead: TRAP positive cells). Scale bar $=50 \mu \mathrm{m}$. (D) The number of TRAP positive cells per linear bone perimeter $(* * * p<0.00 \mathrm{I})$. (E) Representative masson's trichrome-stained images of tooth extraction sockets (black dotted line: tooth extraction sockets of mesial roots). Scale bar $=500 \mu \mathrm{m}$. (F) The area percentage of collagen fibers $(* * * p<0.00 \mathrm{I})$. (G) Representative anti-VEGFA immunohistochemical images of tooth extraction sockets (white arrowhead: VEGFA positive blood vessels). Scale bar=100 $\mu \mathrm{m}$. $(\mathbf{H})$ The number of VEGFA positive blood vessels ( $* * * 0<0.001)$.

Abbreviations: $\mathrm{HE}$, hematoxylin and eosin. $\mathrm{SEV}$-AT, small extracellular vesicles derived from adipose tissue. Zol, zoledronate. Dex, dexamethasone. TRAP, tartrateresistant acid phosphatase. VEGFA, vascular endothelial growth factor $A$. 
A

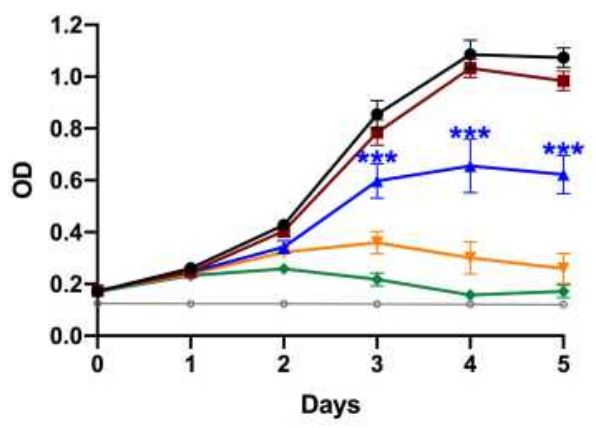

B

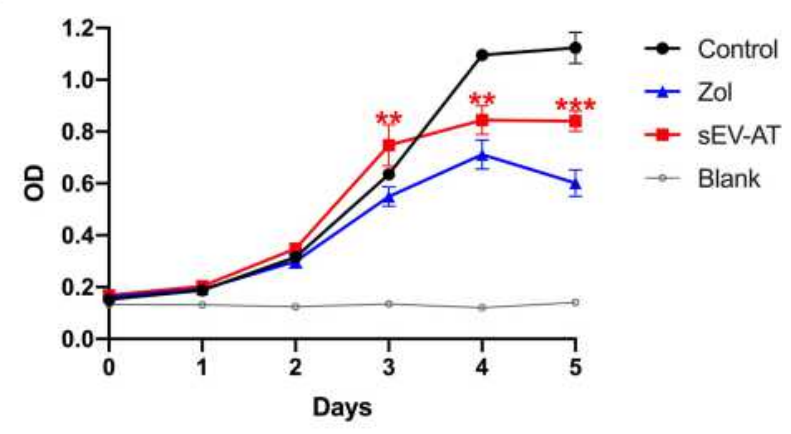

C
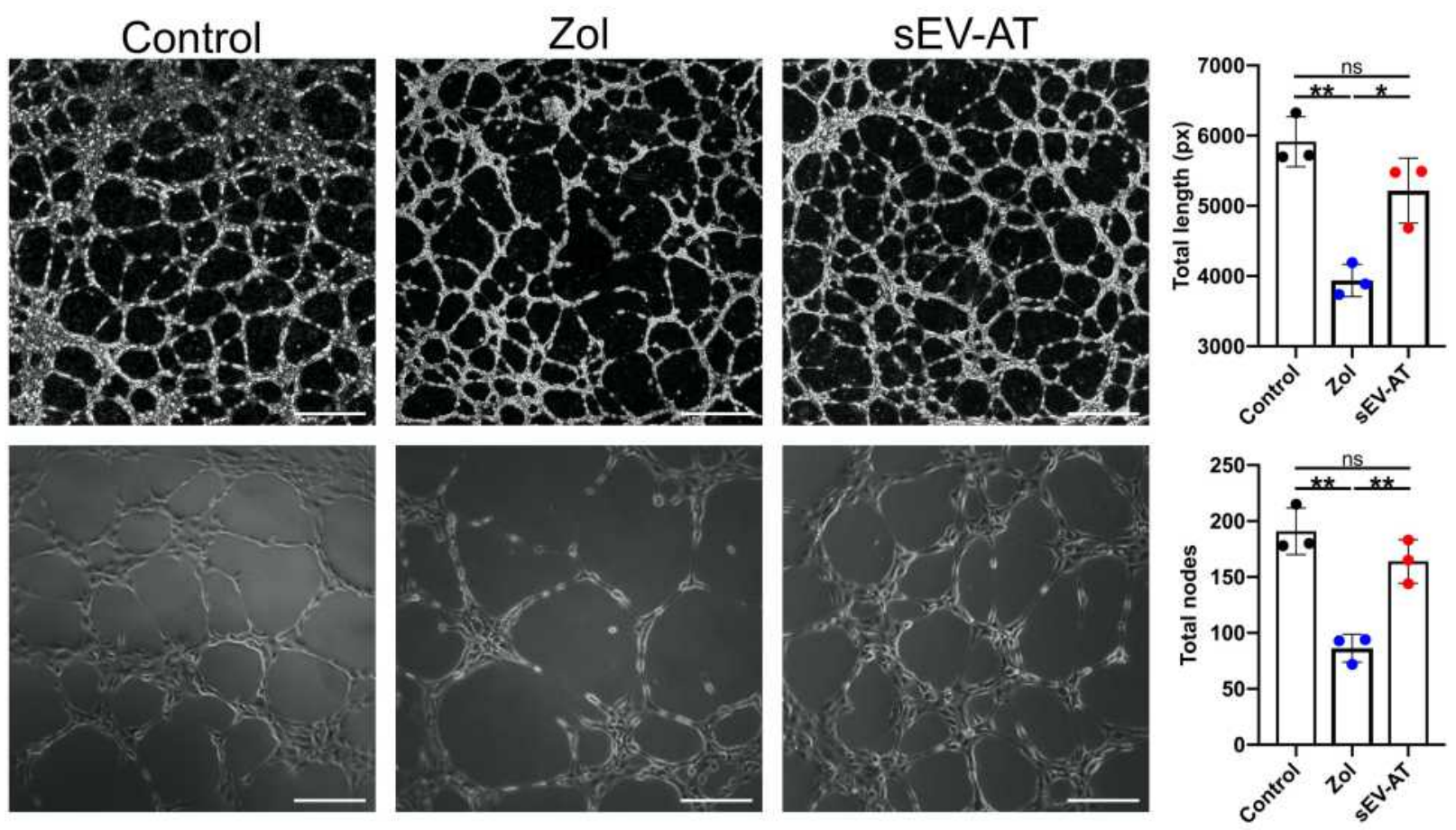

D
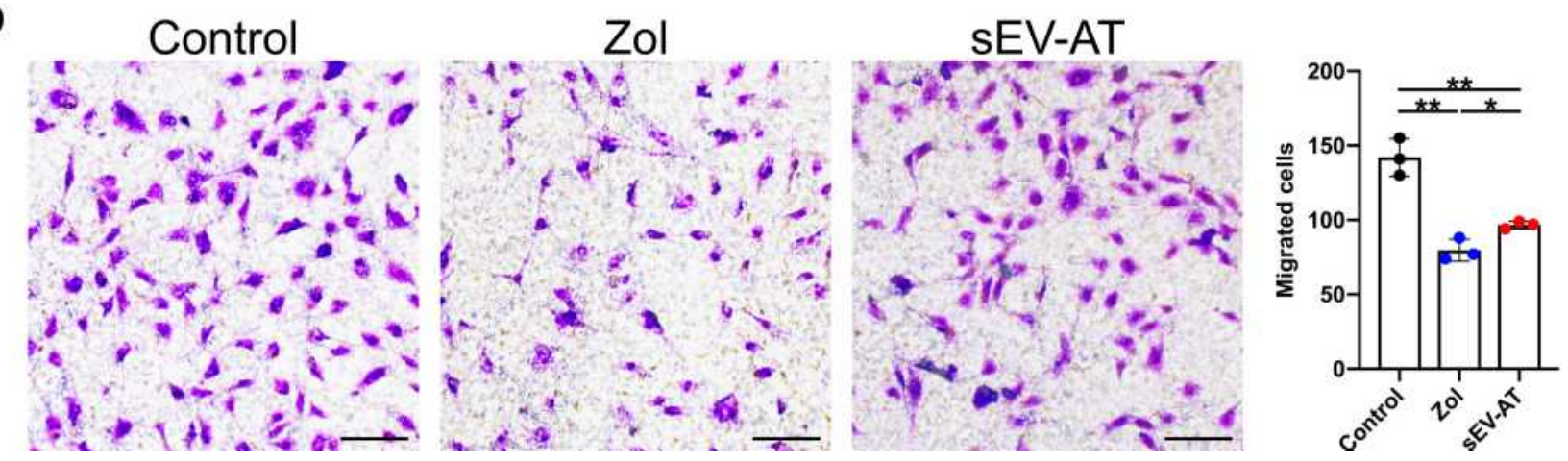

Figure 5 Effects of sEV-AT and Zol on HUVECs. (A) Effects of Zol at different concentration on HUVECs proliferation (***p $<0.00 \mathrm{I})$. (B) Proliferation curves of HUVECs in each group (Control: ECM; Zol: ECM+Zol; sEV-AT: ECM+Zol+sEV-AT) ( ${ }^{* *} p<0.01$, $\left.{ }^{* * *} p<0.00 \mathrm{I}\right)$. (C) Representative tube-like structures of HUVECs in each group. Scale bar=200 $\mu \mathrm{m}$ (upper), scale bar $=500 \mu \mathrm{m}$ (lower). Total length and total nodes of all tubing per field of view from three individual experiments (ns: $P>0.05$, * $P<0.05$, ** $<<0.0 \mathrm{I}$ ). (D) Representative microscope images of migrated HUVECs in each group. Scale bar $=100 \mu \mathrm{m}$. Migrated cells per field of view from three individual experiments $\left({ }^{*} \mathrm{p}<0.05\right.$, $\left.* * 2<0.0 \mathrm{I}\right)$.

Abbreviations: sEV-AT, small extracellular vesicles derived from adipose tissue. HUVECs, human umbilical vein endothelial cells. Zol, zoledronate. ECM, endothelial cell medium. 
human normally takes 2-3 months to heal, while the healing of rats takes much shorter. In our study, we found that all extraction sockets were covered with epithelium completely and filled with newly formed bone in untreated rats within two weeks post tooth extraction. Thus, samples of untreated rats were collected two weeks after tooth extraction as healing control. Considering that healing of extraction sockets takes shorter time in rats than in human, the samples of the Zol+Dex group and the sEV-AT group were collected at 2, 4 weeks post tooth extraction for analysis.

Cell-based therapies have been proved efficiently to prevent BRONJ in several studies. ${ }^{9-11,18,28,29}$ sEV have similar therapeutic effects as their parent cells. Increasing evidence showed that SEV have significant advantages over cell therapy, including easier storage, less risks of malignant transformation, and increasing stability. ${ }^{14,30,31}$ sEV-based therapies are promising alternatives to cellbased therapies. A recent study stated that extracellular vesicles released from mesenchymal stem cells (MSCEVs) could prevent BRONJ. ${ }^{32}$ However, MSC-EVs require much work to be extracted after mass cultivation of MSC. Direct isolation of sEV from adipose tissue, an easily accessible source of biological material, costs less time and money. And it results in higher yields compared to isolation of cell culture derived SEV. Lipoaspirate nanoparticles, consisting of extracellular vesicles and lipoproteins, were proved to have similar anti-inflammatory and protective functions as extracellular vesicles from ADSCs. ${ }^{33}$ Our previous study found the potential of sEVAT on angiogenesis and soft tissue regeneration. ${ }^{16,17}$ Furthermore, 45 conserved miRNAs were enriched in sEV-AT compared to sEV derived from ADSCs according to our previous study. These miRNAs were reported to participate in various functions, such as adipogenesis, angiogenesis, or metabolism. ${ }^{34}$ Secretome derived from an intact adipose environment may function better compared to adipose tissue that had been digested, cultured and lacked certain cell types. In this study, we found that sEV-AT contributed to wound healing and tissue regeneration of tooth extraction sockets which were disturbed by Zol+Dex. To our knowledge, our findings are the first to show that systemic transplantation of sEV-AT could prevent the onset of BRONJ in rats that have received Zol + Dex treatment. Systemic transplantation of sEV-AT could be a potential prevention and treatment strategies for BRONJ.

Angiogenesis is essential for wound healing. Zol was reported to suppress the vascularization after tooth extraction. ${ }^{35}$ We observed that there were less blood vessels in the extraction sockets after Zol+Dex administration. In contrast, sEV-AT administration promoted new blood vessels formation. We further investigated the effects of Zol and sEV-AT on HUVECs. Zol inhibited the proliferation, migration and angiogenesis of HUVECs, which was consistent with previous reports. ${ }^{36}$ sEV-AT protected HUVECs from being inhibited by Zol. These results demonstrated that sEV-AT promoted angiogenesis, which contributed to the prevention of BRONJ. Zoledronate has been reported to impact angiogenesis by disturbing the expression of some important mRNAs and proteins. ${ }^{36-38}$ Our previous study demonstrated that sEV-AT contained miRNAs, such as miR-150-3p, miR-126a-3p, which were associated with angiogenesis. ${ }^{34}$ We will further study on the molecular mechanism of how sEV-AT influence angiogenesis and prevent BRONJ.

However, our current study still has some limitations. The healing of extraction sockets in BRONJ is affected by many factors other than angiogenesis, such as bone marrow stromal cells, osteocytes, osteoclasts, collagen fiber formation. ${ }^{39-43}$ In our study, we found that sEV-AT administration increased osteoclasts, collagen fibers, blood vessels, and reduced empty osteocyte lacunae. Yet the comprehensive effects of sEV-AT were not fully investigated. The effects of sEV-AT on altered microenvironment of maxillofacial bone induced by Zol require further study.

\section{Conclusion}

In this study, we successfully built a BRONJ rat model with Zol and Dex administration, combining tooth extraction. sEV-AT contributed to both osseous and soft tissue regeneration in the BRONJ rat model. sEV-AT promoted the proliferation, migration and angiogenesis of HUVECs inhibited by Zol, which is vital in extraction sockets healing.

In summary, sEV-AT, which are easily obtained, could be a promising biological product to prevent BRONJ.

\section{Author Contributions}

All authors made substantial contributions to conception and design, acquisition of data, or analysis and interpretation of data; took part in drafting the article or revising it critically for important intellectual content; agreed to submit to the current journal; gave final approval for the version to be published; and agreed to be accountable for all aspects of the work. 


\section{Funding}

This work was supported by grants from the National Key Research and Development Program of China (2017YFA0104800) and Key Project of Sichuan province (2019YFS0515).

\section{Disclosure}

The authors report no conflicts of interest in this work.

\section{References}

1. Marx RE. Pamidronate (aredia) and zoledronate (zometa) induced avascular necrosis of the jaws: a growing epidemic. $J$ Oral Maxillofac Surg. 2003;61(9):1115-1117. doi:10.1016/S02782391(03)00720-1

2. Zhang X, Hamadeh I, Song S, et al. Osteonecrosis of the jaw in the United States food and drug administration's adverse event reporting system (faers). J Bone Miner Res. 2016;31(2):336-340. doi:10.1002/ jbmr.2693

3. Ruggiero SL, Dodson TB, Fantasia J, et al. American association of oral and maxillofacial surgeons position paper on medication-related osteonecrosis of the jaw-2014 update. J Oral Maxillofac Surg. 2014;72(10):1938-1956. doi:10.1016/j.joms.2014.04.031

4. Chang J, Hakam A, McCauley L. Current understanding of the pathophysiology of osteonecrosis of the jaw. Curr Osteoporos Rep. 2018;16(5):584-595. doi:10.1007/s11914-018-0474-4

5. Ramaglia L, Guida A, Iorio-Siciliano V, Cuozzo A, Blasi A, Sculean A. Stage-specific therapeutic strategies of medication-related osteonecrosis of the jaws: a systematic review and meta-analysis of the drug suspension protocol. Clin Oral Investig. 2018;22(2):597-615. doi:10.1007/s00784-017-2325-6

6. Hayashida S, Soutome S, Yanamoto S, et al. Evaluation of the treatment strategies for medication-related osteonecrosis of the jaws (mronj) and the factors affecting treatment outcome: a multicenter retrospective study with propensity score matching analysis. $J$ Bone Miner Res. 2017;32(10):2022-2029. doi:10.1002/jbmr.3191

7. Wensveen F, Valentić S, Šestan M, Wensveen T, Polić B. Interactions between adipose tissue and the immune system in health and malnutrition. Semin Immunol. 2015;27(5):322-333. doi:10.1016/j. smim.2015.10.006

8. Yang Z, Wei Z, Wu X, Yang H. Screening of exosomal miRNAs derived from subcutaneous and visceral adipose tissues: determination of targets for the treatment of obesity and associated metabolic disorders. Mol Med Rep. 2018;18(3):3314-3324. doi:10.3892/ mmr.2018.9312

9. Barba-Recreo P, Del Castillo Pardo de Vera JL, Georgiev-Hristov T. Adipose-derived stem cells and platelet-rich plasma for preventive treatment of bisphosphonate-related osteonecrosis of the jaw in a murine model. J Craniomaxillofac Surg. 2015;43(7):1161-1168. doi:10.1016/j.jcms.2015.04.026

10. Zang X, He L, Zhao L, He Y, Xiao E, Zhang Y. Adipose-derived stem cells prevent the onset of bisphosphonate-related osteonecrosis of the jaw through transforming growth factor beta-1-mediated gingival wound healing. Stem Cell Res Ther. 2019;10(1):169. doi:10.1186/ s13287-019-1277-y

11. Kuroshima S, Sasaki M, Nakajima K, Tamaki S, Hayano H, Sawase T. Transplantation of noncultured stromal vascular fraction cells of adipose tissue ameliorates osteonecrosis of the jaw-like lesions in mice. $J$ Bone Miner Res. 2018;33(1):154-166. doi:10.1002/jbmr.3292

12. Karnoub A, Dash A, Vo A, et al. Mesenchymal stem cells within tumour stroma promote breast cancer metastasis. Nature. 2007;449 (7162):557-563. doi:10.1038/nature06188
13. Comella K, Parcero J, Bansal H, et al. Effects of the intramyocardial implantation of stromal vascular fraction in patients with chronic ischemic cardiomyopathy. $J$ Transl Med. 2016;14(1):158. doi:10.1186/s12967-016-0918-5

14. Bateman M, Strong A, Gimble J, Bunnell B. Concise review: using fat to fight disease: a systematic review of nonhomologous adipose-derived stromal/stem cell therapies. Stem Cells. 2018;36 (9):1311-1328. doi:10.1002/stem.2847

15. Mathieu M, Martin-Jaular L, Lavieu G, Théry C. Specificities of secretion and uptake of exosomes and other extracellular vesicles for cell-to-cell communication. Nat Cell Biol. 2019;21(1):9-17. doi:10.1038/s41556-018-0250-9

16. Dai M, Yu M, Zhang Y, Tian W. Exosome-like vesicles derived from adipose tissue provide biochemical cues for adipose tissue regeneration. Tissue Eng Part A. 2017;23(21-22):1221-1230. doi:10.1089/ten.tea.2017.0045

17. Dong J, Wu Y, Zhang Y, Yu M, Tian W. Comparison of the therapeutic effect of allogeneic and xenogeneic small extracellular vesicles in soft tissue repair. Int J Nanomedicine. 2020;15:6975-6991. doi:10.2147/IJN.S269069

18. Kaibuchi N, Iwata T, Yamato M, Okano T, Ando T. Multipotent mesenchymal stromal cell sheet therapy for bisphosphonate-related osteonecrosis of the jaw in a rat model. Acta Biomater. 2016;42:400-410. doi:10.1016/j.actbio.2016.06.022

19. Kuroshima S, Nakajima K, Sasaki M, et al. Systemic administration of quality- and quantity-controlled pbmncs reduces bisphosphonate-related osteonecrosis of jaw-like lesions in mice. Stem Cell Res Ther. 2019;10(1):209. doi:10.1186/s13287-019-1308-8

20. Polchow B, Kebbel K, Schmiedeknecht G, et al. Cryopreservation of human vascular umbilical cord cells under good manufacturing practice conditions for future cell banks. J Transl Med. 2012;10(1):98. doi:10.1186/1479-5876-10-98

21. Jackson G, Morgan G, Davies F, et al. Osteonecrosis of the jaw and renal safety in patients with newly diagnosed multiple myeloma: medical research council myeloma ix study results. $\mathrm{Br} J$ Haematol. 2014;166(1):109-117. doi:10.1111/bjh.12861

22. Gralow J, Barlow W, Paterson A, et al. Phase III randomized trial of bisphosphonates as adjuvant therapy in breast cancer: S0307. J Natl Cancer Inst. 2020;112(7):698-707. doi:10.1093/jnci/djz215

23. Rosini S, Rosini S, Bertoldi I, Frediani B. Understanding bisphosphonates and osteonecrosis of the jaw: uses and risks. Eur Rev Med Pharmacol Sci. 2015;19(17):3309-3317.

24. Hasegawa T, Hayashida S, Kondo E, et al. Medication-related osteonecrosis of the jaw after tooth extraction in cancer patients: a multicenter retrospective study. Osteoporosis Int. 2019;30 (1):231-239. doi:10.1007/s00198-018-4746-8

25. Otto S, Pautke C, Van den Wyngaert T, Niepel D, Schiødt M. Medication-related osteonecrosis of the jaw: prevention, diagnosis and management in patients with cancer and bone metastases. Cancer Treat Rev. 2018;69:177-187. doi:10.1016/j.ctrv.2018.06.007

26. Jabbour Z, El-Hakim M, Henderson J, de Albuquerque R. Bisphosphonates inhibit bone remodeling in the jaw bones of rats and delay healing following tooth extractions. Oral Oncol. 2014;50 (5):485-490. doi:10.1016/j.oraloncology.2014.02.013

27. Ogata K, Katagiri W, Osugi M, et al. Evaluation of the therapeutic effects of conditioned media from mesenchymal stem cells in a rat bisphosphonate-related osteonecrosis of the jaw-like model. Bone. 2015;74:95-105. doi:10.1016/j.bone.2015.01.011

28. Li Y, Xu J, Mao L, et al. Allogeneic mesenchymal stem cell therapy for bisphosphonate-related jaw osteonecrosis in swine. Stem Cells Dev. 2013;22(14):2047-2056. doi:10.1089/scd.2012.0615

29. Kikuiri T, Kim I, Yamaza T, et al. Cell-based immunotherapy with mesenchymal stem cells cures bisphosphonate-related osteonecrosis of the jaw-like disease in mice. J Bone Miner Res. 2010;25 (7):1668-1679. doi:10.1002/jbmr.37 
30. Cai Y, Li J, Jia C, He Y, Deng C. Therapeutic applications of adipose cell-free derivatives: a review. Stem Cell Res Ther. 2020;11(1):312. doi:10.1186/s13287-020-01831-3

31. Phinney D, Pittenger M. Concise review: msc-derived exosomes for cell-free therapy. Stem Cells. 2017;35(4):851-858. doi:10.1002/ stem. 2575

32. Watanabe J, Sakai K, Urata Y, Toyama N, Nakamichi E, Hibi H. Extracellular vesicles of stem cells to prevent bronj. J Dent Res. 2020;99(5):552-560. doi:10.1177/0022034520906793

33. Tian M, Ticer T, Wang Q, et al. Adipose-derived biogenic nanoparticles for suppression of inflammation. Small. 2020;16(10):e1904064. doi:10.1002/smll.201904064

34. Zhang Y, Yu M, Dai M, et al. Mir-450a-5p within rat adipose tissue exosome-like vesicles promotes adipogenic differentiation by targeting wisp2. J Cell Sci. 2017;130(6):1158-1168. doi:10.1242/ jcs. 197764

35. Kün-Darbois J, Libouban H, Mabilleau G, Pascaretti-Grizon F, Chappard D. Bone mineralization and vascularization in bisphosphonate-related osteonecrosis of the jaw: an experimental study in the rat. Clin Oral Investig. 2018;22(9):2997-3006. doi:10.1007/s00784-018-2385-2

36. Kim B, Yang S, Kim C, Lee J. Zoledronate suppresses VEGF-induced capillary tube formation and inhibits expression of interferon-induced transmembrane protein-1 in human umbilical vein endothelial cells. Int J Mol Med. 2018;41(5):2879-2884. doi:10.3892/ijmm.2018.3497
37. Wehrhan F, Amann K, Möbius P, et al. Bronj-related jaw bone is associated with increased $\mathrm{dlx}-5$ and suppressed osteopontin-implication in the site-specific alteration of angiogenesis and bone turnover by bisphosphonates. Clin Oral Investig. 2015;19 (6):1289-1298. doi:10.1007/s00784-014-1354-7

38. Ohlrich EJ, Coates DE, Cullinan MP, et al. The bisphosphonate zoledronic acid regulates key angiogenesis-related genes in primary human gingival fibroblasts. Arch Oral Biol. 2016;63:7-14. doi:10.1016/j.archoralbio.2015.11.013

39. Pazianas M, van der Geest S, Miller P. Bisphosphonates and bone quality. Bonekey Rep. 2014;3:529. doi:10.1038/bonekey.2014.24

40. Gong X, Yu W, Zhao H, Su J, Sheng Q. Skeletal site-specific effects of zoledronate on in vivo bone remodeling and in vitro bmscs osteogenic activity. Sci Rep. 2017;7(1):36129. doi:10.1038/srep36129

41. Yu Y, Lieu S, Hu D, Miclau T, Colnot C. Site specific effects of zoledronic acid during tibial and mandibular fracture repair. PLoS One. 2012;7(2):e31771. doi:10.1371/journal.pone.0031771

42. Taniguchi N, Osaki M, Onuma K, et al. Bisphosphonate-induced reactive oxygen species inhibit proliferation and migration of oral fibroblasts: a pathogenesis of bisphosphonate-related osteonecrosis of the jaw. J Periodontol. 2020;91(7):947-955. doi:10.1002/JPER.190385

43. Su J, Feng M, Han W, Zhao H. The effects of bisphosphonate on the remodeling of different irregular bones in mice. J Oral Pathol Med. 2015;44(8):638-648. doi:10.1111/jop.12281
International Journal of Nanomedicine

\section{Publish your work in this journal}

The International Journal of Nanomedicine is an international, peerreviewed journal focusing on the application of nanotechnology in diagnostics, therapeutics, and drug delivery systems throughout the biomedical field. This journal is indexed on PubMed Central, MedLine, CAS, SciSearch ${ }^{\mathbb{}}$, Current Contents ${ }^{\mathbb{R}} /$ Clinical Medicine, $^{2}$

\section{Dovepress}

Journal Citation Reports/Science Edition, EMBase, Scopus and the Elsevier Bibliographic databases. The manuscript management system is completely online and includes a very quick and fair peer-review system, which is all easy to use. Visit http://www.dovepress.com/ testimonials.php to read real quotes from published authors. 